\title{
Design and testing of a 7.8 GHz power extractor using a cylindrical dielectric-loaded waveguide
}

\author{
F. Gao, ${ }^{1,2}$ M. E. Conde, ${ }^{1}$ W. Gai, ${ }^{1}$ C. Jing, ${ }^{3}$ R. Konecny, ${ }^{1}$ W. Liu,,${ }^{1}$ J. G. Power, ${ }^{1}$ T. Wong,,${ }^{2}$ and Z. Yusof ${ }^{1}$ \\ ${ }^{1}$ Argonne National Laboratory, Argonne, Illinois 60439, USA \\ ${ }^{2}$ Illinois Institute of Technology, Chicago, Illinois 60616, USA \\ ${ }^{3}$ Euclid TechLabs, LLC, Solon, Ohio 44139, USA \\ (Received 30 January 2008; published 1 April 2008)
}

\begin{abstract}
Dielectric-loaded power extraction is a method for the generation of high-power radio frequency (rf) waves under development for future particle accelerators. In this method, a high-charge electron beam drives a wakefield in a dielectric-loaded waveguide (the decelerator) and an rf output coupler extracts the rf power into an external waveguide. We report on the experimental demonstration of a $7.8 \mathrm{GHz}$ dielectricloaded power extractor at the Argonne Wakefield Accelerator facility. We have generated more than $30 \mathrm{MW}$ of $\mathrm{rf}$ power with a pulse length of approximately $1.7 \mathrm{~ns}$ by passing a single $66 \mathrm{nC}$ electron bunch through the power extractor. We have also used a train of 4 electron bunches to show a clear signature of field superposition. Test results are in good agreement with predictions.
\end{abstract}

DOI: 10.1103/PhysRevSTAB.11.041301

PACS numbers: 41.75.Lx, 41.60.Bq, 84.40.-x

\section{INTRODUCTION}

One potential way to increase the energy of future particle accelerators beyond the existing LHC [1] and proposed ILC [2] is to use the two-beam acceleration (TBA) concept $[3,4]$. The TBA scheme can be broken down into three stages: (i) radio frequency (rf) power is extracted from a low-energy, high-current drive bunch by a decelerator; (ii) rf power is coupled out of the decelerator and into a transfer waveguide; and (iii) rf power is delivered into an accelerator for acceleration of the high-energy, low-current main bunch. This option may overcome some of the limitations of high-power rf generation, transfer, and pulse compression compared to a klystron-based system $[4,5]$ at frequencies above X-band [6] and power levels beyond a couple of hundred megawatts [7]. This is due to the ease with which the rf power characteristics can be changed by manipulating the decelerator and its drive bunch. It has been demonstrated that a properly designed TBA scheme can provide rf power on the order of gigawatts [8].

There is an ongoing program at Argonne National Laboratory to develop dielectric-TBA. This uses a dielectric-loaded waveguide for both the decelerator and accelerator [9,10]. As shown in Fig. 1, the dielectric-loaded waveguide consists of a cylindrical metallic tube filled with dielectric material in the region $a \leq r \leq b$, and a vacuum channel in the region $0 \leq r<a$ for the bunch. The structure is designed so that the $\mathrm{TM}_{01}$ mode has the strongest interaction with an on-axis bunch. By changing the inner and outer radii, and the permittivity of the dielectric, the structure can be tuned so that the phase velocity is set to the particle velocity at the desired frequency. Compared with the traditional, iris-loaded metallic waveguide of similar rf properties, the dielectric-loaded waveguide is potentially simpler to fabricate due to its uniformity in the longitudinal direction, especially when high precision is needed at high frequencies [11]. Furthermore, a simple scheme is available for damping undesired deflection modes [12].

In this paper, we report on a proof of principle experimental test of a broadband, efficiently coupled $7.8 \mathrm{GHz}$ power extractor driven by an ultrarelativistic beam; the Argonne Wakefield Accelerator (AWA) high-current beam. We define the term dielectric power extractor to refer to the combination of the decelerator (a dielectricloaded waveguide) and the rf output coupler, as shown in Fig. 2. The use of a dielectric-loaded waveguide as part of an rf power extractor was first proposed in 1991 [13] and later, a $7.8 \mathrm{GHz}$ structure shows $3.4 \mathrm{MW}$ of power was generated by a $24 \mathrm{nC}$ single bunch [14], and also a $21 \mathrm{GHz}$ structure was tested at the CTF2 [15].

The remainder of the article is organized as follows. In Sec. II, the theoretical background for the cylindrical dielectric-loaded waveguide is introduced. In Secs. III and IV, the simulations and cold tests are shown. In Sec. V, the detailed experiment setup is presented and both bench and beam testing results are shown. Finally, we give a summary and future plan in Sec. VI.

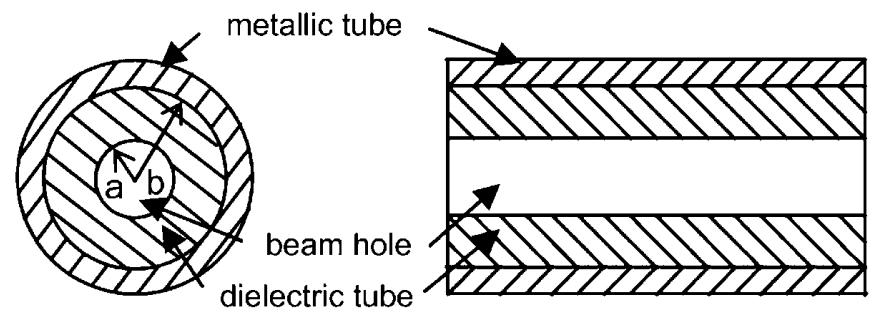

FIG. 1. Transverse and longitudinal cross-sectional views of the circular dielectric-loaded waveguide. 


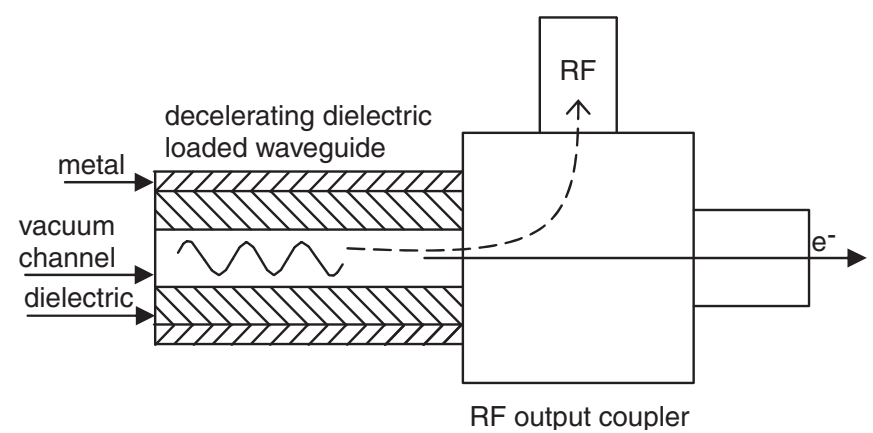

FIG. 2. Dielectric-loaded power extractor consists of a dielectric-loaded decelerator and an rf output coupler.

\section{BEAM-STRUCTURE INTERACTION IN A DIELECTRIC-LOADED WAVEGUIDE}

In this section, the rf power level and rf pulse duration excited by a drive bunch (or bunch train) in a dielectric loaded waveguide are described.

\section{A. General relationships for the traveling wave waveguide}

We will need to make use of two general relationships of the traveling wave (TW) waveguide so we begin by recalling them here for the convenience of the reader [Wangler [16]]. For each mode in the structure, the relationship between the longitudinal accelerating field amplitude, $E_{a}$, and the stored energy per unit length, $U$, are related through the accelerator figure of merit loss factor per unit length

$$
k_{L}=\frac{E_{a}^{2}}{4 U}
$$

The $k_{L}$ of each mode can be calculated with standard electromagnetic codes such as CST MICROWAVE STUDIO [17]. The TW power, $P_{w}$, is related to $E_{a}$ through

$$
P_{w}=\frac{E_{a}{ }^{2} v_{g}}{4 k_{L}}
$$

where $v_{g}$ is the group velocity of the TW mode.

\section{B. The beam driven traveling wave waveguide}

Consider a charged particle beam (the drive bunch) traveling down the axis of a dielectric-loaded waveguide (the decelerator) of the length $L$. The drive bunch will excite a wakefield that carries a TW power, $P_{w}$, into the modes of the structure. In this article, the drive bunch (or drive-bunch train) is considered ultrarelativistic (the particle velocity equals $c$, the speed of light in free space).

\section{Time structure of the rf pulse}

Let a drive bunch traveling in the $z+$ direction enter the decelerator of length $L$ at the moment $t=0$ and location $z=0$ [Fig. 3(a)]. At time $t$ when the bunch is still inside the decelerator [Fig. 3(b)], the head of the rf pulse (moving

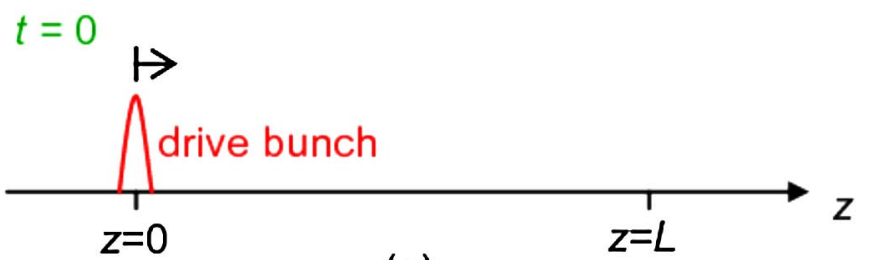

(a)
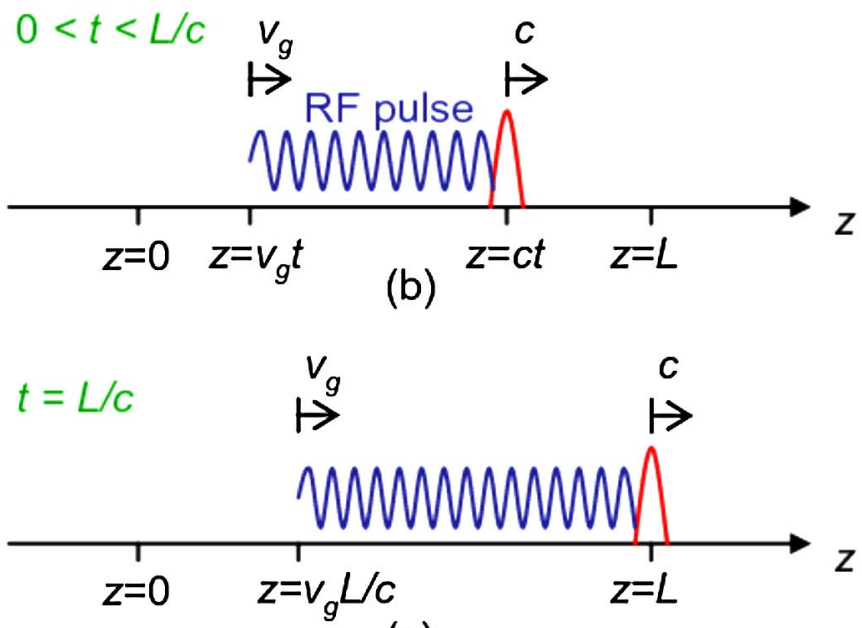

(c)

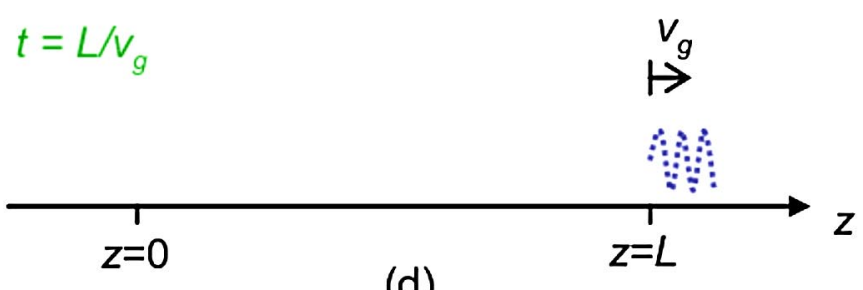

(d)

FIG. 3. (Color) The rf pulse due to a single drive bunch. Snapshots of a single bunch and the excited rf pulse inside the decelerator region $0 \leq z \leq L$. (a) The bunch enters the decelerator $(t=0)$; (b) the bunch and the rf pulse are fully inside the decelerator where the head and the tail of the rf pulse travel at different speed $(0<t<L / c)$; (c) the bunch reaches the exit of the decelerator $(t=L / c)$; (d) the tail of the rf pulse exits the decelerator $\left(t=L / v_{g}\right)$.

at $c$ ) is located at $z=c t$, while the tail of the rf pulse (moving at $v_{g}$ ) is located at $z=v_{g} t$. When the bunch (and the head of the rf pulse) reaches the downstream end $(z=$ $L$ ) of the decelerator [Fig. 3(c)], the time is $t=L / c$ and the tail of the rf pulse has reached the position $z=v_{g} L / c$. At this moment, the generated rf pulse would begin to be extracted by an $\mathrm{rf}$ coupler (not shown) at $z=L$, but in this heuristic description we imagine the rf packet propagating out of the end of the decelerator at $z=L$. The final event occurs when the tail of the rf pulse exits the decelerator [Fig. 3(d)] at time $t=L / v_{g}$. The duration of the rf pulse is the difference between the moment the bunch reaches the exit $(t=L / c)$ and the moment the tail of the rf pulse reaches the exit $\left(t=v_{g} L / c\right)$. Therefore, the rf pulse dura- 
tion for single-bunch excitation is given by

$$
\tau_{s}=L\left(1-\beta_{g}\right) / v_{g},
$$

where $\beta_{g}=v_{g} / c$ is the relativistic group velocity.

Now let us consider the field buildup process due to bunch-train excitation. If a second bunch reaches the exit after the first bunch but before the tail of the first rf pulse, then there will be a region where the rf pulses of the two bunches overlap. To create constructive interference (inphase superposition) of the excited rf fields, the frequency of the excited rf mode $\left(f_{0}\right)$ is chosen to be a harmonic of the bunch frequency, $1 / T_{b}$, for a train of $n$ bunches evenly spaced in time by $T_{b}$.

The buildup in time of the amplitude of the longitudinal electrical field due to a bunch train $\left(E_{a t}\right)$ at the exit of the decelerator $(z=L)$ is shown in Fig. 4. (Note: This is the field that would be observed by an antenna located at $z=L$ and we have now redefined time so that $t=0$ corresponds to arrival of the first bunch at $z=L$.) The rf pulses excited by individual bunches are labeled in color and the increasing amplitude of the net field $\left(E_{a t}\right)$ is due to superposition. The time structure of the bunch-train $\mathrm{rf}$ pulse (at $z=L$ ) consists of a rise time, a flattop, and a fall time; all of which are determined by the overlap of the individual single-bunch rf pulses. The rise time is determined by the number of bunches whose rf pulses overlap with the first rf pulse and is equal to ceiling $\left(\tau_{s} / T_{b}\right)-1$. For example, if the single-bunch pulse length is $\tau_{s}=$ $2.4 \mathrm{~ns}$ and the bunches are spaced at $T_{b}=0.769 \mathrm{~ns}$, then there will be ceiling $(2.4 / 0.769)-1=3$ trailing bunches whose rf pulses overlap with the first rf pulse. In general, the number of rf pulses that overlap at the exit of the decelerator is $N=$ ceiling $\left(\tau_{s} / T_{b}\right)$. From Fig. 4 , it can be seen for a long bunch train with $n \geq N$, the rise time $t_{r}$ can be written as

$$
t_{r}=(N-1) \times T_{b} .
$$

Similarly the fall time is

$$
t_{f}=t_{r}
$$

The steady-state (flattop) duration of the rf pulse is simply

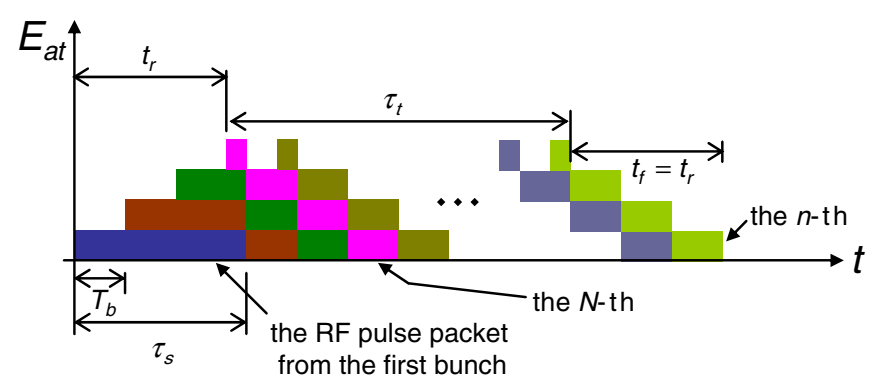

FIG. 4. (Color) The rf pulse due to a drive-bunch train. The longitudinal electrical field amplitude experienced at the exit of the decelerator with bunch train excitation (pulses excited by different bunches are labeled with different colors). the total length duration minus the rise time and the fall time, i.e.

$$
\tau_{t}=(n-1) \times T_{b}+\tau_{s}-2 t_{r} .
$$

Note that for a very long train $(n \gg N)$, the pulse length is simply $\tau_{t} \approx n T_{b}$.

\section{Power levels}

In this subsection, we provide expressions for the TW power excited by a single drive bunch, $P_{s}$, and a train of drive bunches, $P_{t}$. The derivations are given in the Appendix.

For a single drive bunch on axis in a lossless (the attenuation coefficient $\alpha_{0}=0$ ) decelerator of length $L$, the power level excited, $P_{s}$, for a given structure mode is $[18,19]$

$$
P_{s}=q^{2} k_{L} v_{g}\left(\frac{1}{1-\beta_{g}}\right)^{2} \Phi^{2} \quad\left(0 \leq t \leq \tau_{s}\right)
$$

where $q$ is the bunch charge and $\Phi$, the bunch form factor, is the magnitude of the Fourier transform of the charge distribution function at the mode frequency. It can be shown that, for a Gaussian bunch with an rms length $\sigma_{z}$, the bunch form factor is

$$
\Phi=\exp \left[-\left(k_{z} \sigma_{z}\right)^{2} / 2\right],
$$

where $k_{z}$ is the longitudinal wave number of the deceleration mode. Structure attenuation can be taken into account by modifying Eq. (5) as follows:

$$
P_{s}(t)=q^{2} k_{L} v_{g}\left(\frac{1}{1-\beta_{g}}\right)^{2} \Phi^{2} e^{-2 \alpha_{0} v_{g} t} \quad\left(0 \leq t \leq \tau_{s}\right),
$$

where $t=0$ is the moment the bunch exits the decelerator, i.e., the moment the head of the excited rf pulse exits the decelerator.

Similarly, for a long bunch train with $n \geq N$, traveling on axis in a lossless structure the TW power, $P_{t}$, generated will reach a steady-state value of

$$
P_{t}=q^{2} \frac{k_{L}}{v_{g}}\left(\frac{L}{T_{b}}\right)^{2} \Phi^{2} .
$$

From Eq. (5) and (8), it can be easily shown that

$$
P_{t}=\left(\frac{\tau_{s}}{T_{b}}\right)^{2} P_{s}
$$

where we remind the reader that $n \geq N$. The lower and upper limits of this power level are given by

$$
(N-1)^{2} P_{s}<P_{t} \leq N^{2} P_{s} .
$$

Again, if the structure is lossy, the traveling power $P_{t}$ is modified as follows:

$$
P_{t}=q^{2} \frac{k_{L}}{v_{g}}\left(\frac{1-e^{-\alpha_{0} L}}{\alpha_{0} T_{b}}\right)^{2} \Phi^{2} .
$$




\section{POWER-EXTRACTOR DESIGN AND SIMULATION}

Efficient $\mathrm{rf}$ power generation first requires the drivebunch energy to be efficiently coupled into the desired decelerator mode (typically $\mathrm{TM}_{01}$ ) and then, that the $\mathrm{rf}$ output coupler efficiently extracts this mode power into the output waveguide. In this section, the design and simulation of the decelerator and rf output coupler are described.

\section{A. rf power generation in the dielectric-loaded decelerator}

From inspection of Eqs. (7) and (11), it is seen that high rf power is generated when several decelerator design constraints are met: (i) the inner radius, $a$, should be large to allow for high beam-charge transmission but small to keep $k_{L}$ high; (ii) the length, $L$, should be long to increase the field superposition in multibunch excitation; (iii) the group velocity $v_{g}$ should be small in multibunch excitation for stronger field superposition but also large to reduce the rise time; (iv) the attenuation coefficient should be small to reduce loss. In other words, the final design is a tradeoff between these requirements.

CST MAFIA T2 solver [20] is used to calculate the excited gradients $E_{a s}$ by a single bunch and $E_{a t}$ by a bunch train, respectively, from which we can calculate the TW power inside the structure. In the simulations, a Gaussian electron bunch with bunch length $\sigma_{z}=2 \mathrm{~mm}$ (typical for bunches used during the beam test) is used, and the bunch charge is set to $1 \mathrm{nC}$ for scaling purposes, while in the experiment the expected charge range is $1-100 \mathrm{nC}$. The dielectric material is Cordierite with a relative permittivity 4.6.

The final optimized parameters of the structure for generating high TW power $P_{t}$ are listed in Table I. The frequency of the excited $\mathrm{TM}_{01}$ mode was chosen to be $7.8 \mathrm{GHz}$, which is the 6th harmonic of the bunch frequency $1.3 \mathrm{GHz}$. For this proof of principle test, $7.8 \mathrm{GHz}$ gives a large bunch form factor $(\Phi=0.95)$, also it does not require high precision in fabrication. For single-bunch excitation, the longitudinal electrical field excited in the
7.8 GHz peak is plotted in Fig. 5(a), where it can be seen that the rf pulse length is $2.2 \mathrm{~ns}$, while the pulse length given by Eq. (3) is $2.9 \mathrm{~ns}$. The difference is due to end effects at the upstream side of the decelerating waveguide, where a cutoff plug is used to secure the dielectric tube.

For a bunch train, the buildup of the longitudinal electric field excited by 10 consecutive bunches is shown in Fig. 5(b). Close inspection of the simulated fields shows that the field reaches saturation after 4 pulses, with a rise time of $2.3 \mathrm{~ns}$, a pulse length (flattop) of $5.0 \mathrm{~ns}$, and a fall time of $2.3 \mathrm{~ns}$. While the rise time and the fall time agree with the values given by Eqs. (4a) and (4b), the pulse length is slightly different than the $5.2 \mathrm{~ns}$ calculated with Eq. (4c); again due to end effects. The rf pulse will increase in length as more bunches are added, but will not increase in power because there will be no overlap of rf pulses generated from bunches that are far apart. The simulated gradients and power levels listed in Table II are seen to be in good agreement with the results from Eqs. (7) and (11). In addition, we emphasize that the power is proportional to the square of charge as expected from Eq. (7) and (11).

\section{B. Power extraction with the rf coupler}

In order to extract the rf power from the decelerator and into the WR112 rectangular output waveguide, a $\mathrm{TM}_{01}-\mathrm{TE}_{10}$ rf output coupler with a center frequency 7.8 $\mathrm{GHz}$ is needed. In order that very short rf pulses ( $\sim 10 \mathrm{~ns}$ for some future dielectric-based accelerator concepts) can be extracted out, the desired bandwidth (in which the insertion loss is better than $-3 \mathrm{~dB}$ ) is more than 200 MHz. As shown in Figs. 6(a) and 6(b), the downstream end of the dielectric tube (outer radius $b$ ) is inserted into a larger metallic tube (inner radius $d$ ) which is connected to a stepped rectangular waveguide for impedance matching. The downstream metallic beam hole has the same radius as the inner radius of the dielectric, $a$, which is below cutoff for the $\mathrm{TM}_{01}$ mode at $7.8 \mathrm{GHz}$, thus no rf can be coupled into beam hole.

The rf coupler was modeled and optimized to achieve efficient coupling with CST MICROWAVE STUDIO [17]. By changing the cylinder length $h$, the cylinder radius $d$, the

TABLE I. Design parameters of the dielectric-loaded decelerator.

\begin{tabular}{lcc}
\hline \hline rf frequency & $f_{0}$ & $7.8 \mathrm{GHz}$ \\
Inner radius of the dielectric tube & $a$ & $6.02 \mathrm{~mm}$ \\
Outer radius of the dielectric tube & $b$ & $11.17 \mathrm{~mm}$ \\
Dielectric tube length & $L$ & $266 \mathrm{~mm}$ \\
Relative permittivity of the dielectric & $\epsilon_{r}$ & 4.6 \\
Relativistic group velocity of the rf mode & $\beta_{g}$ & 0.23 \\
Loss tangent of the ceramic & $\delta_{d}$ & $5 \times 10^{-4}$ \\
Wall quality factor (metallic wall losses only) & $Q_{w}$ & 8777 \\
Total quality factor (metallic wall and dielectric losses) & $Q$ & 2745 \\
Loss factor per unit length of the structure & $k_{L}$ & $7.46 \times 10^{13} \mathrm{~V}^{2} /(\mathrm{J} \cdot \mathrm{m})$ \\
" $r$ over $Q "$ per unit length of the structure & {$[r / Q]$} & $6.09 \mathrm{~K} \Omega / \mathrm{m}$ \\
\hline \hline
\end{tabular}




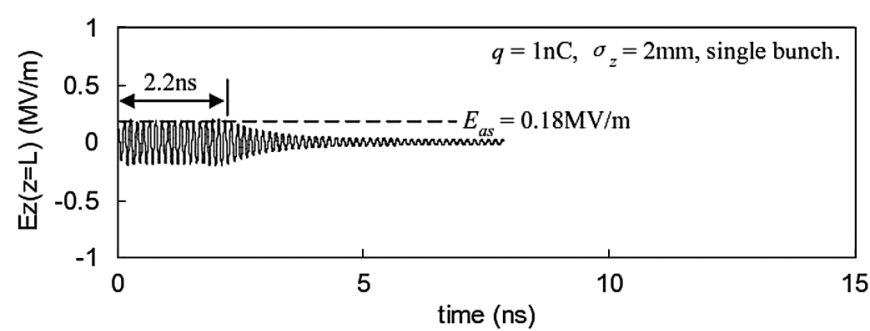

(a)

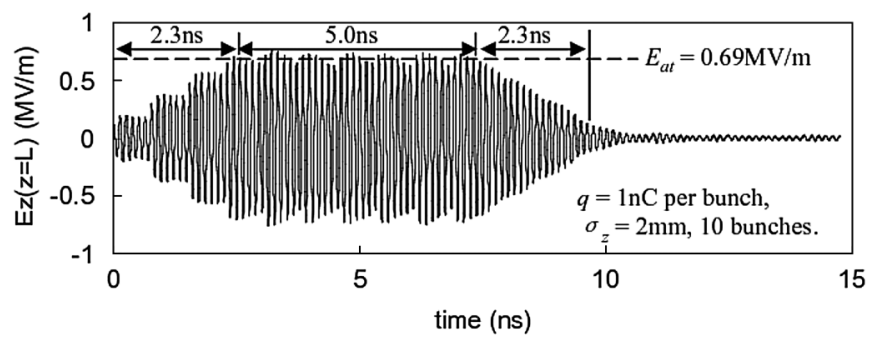

(b)

FIG. 5. MAFIA simulation of the gradient at the end of the decelerator induced by (a) a single bunch; (b) a train of 10 bunches.

TABLE II. Gradient and power generated in the structure of Table I and driven by a single Gaussian bunch and a bunch train $\left(\sigma_{z}=2 \mathrm{~mm}\right)$.

\begin{tabular}{lcccc}
\hline \hline & \multicolumn{3}{c}{ Single bunch } & \multicolumn{2}{c}{ Bunch train } \\
& $E_{a s} \propto q$ & $P_{s} \propto q^{2}$ & $E_{a t} \propto q$ & $P_{t} \propto q^{2}$ \\
\hline$q=1 \mathrm{nC}$ & $0.18 \mathrm{MV} / \mathrm{m}$ & $0.0079 \mathrm{MW}$ & $0.69 \mathrm{MV} / \mathrm{m}$ & $0.11 \mathrm{MW}$ \\
$q=100 \mathrm{nC}$ & $18.0 \mathrm{MV} / \mathrm{m}$ & $79 \mathrm{MW}$ & $69 \mathrm{MV} / \mathrm{m}$ & $1.1 \mathrm{GW}$ \\
\hline \hline
\end{tabular}

width of the narrowed waveguide $w$, and the position of the step $g$, the S-parameters of the coupler was tuned to maximize the coupler bandwidth around the center frequency at $7.8 \mathrm{GHz}$, where the $S_{21}$ is $-0.23 \mathrm{~dB}$, while the $S_{11}$ is $-12.9 \mathrm{~dB}$. From 7.71 to $7.94 \mathrm{GHz}$, the $S_{21}$ is better than $-1 \mathrm{~dB}$, and from 7.68 to $8.06 \mathrm{GHz}$ the $\mathrm{S}_{21}$ is better than $-3 \mathrm{~dB}$.

\section{FABRICATION AND COLD TEST}

In this section, the fabrication and low-power rf measurement (cold test) of the power-extractor assembly, including the decelerator and the rf coupler, are described. Based on the above numerical simulations, the assembly was fabricated at the Argonne National Laboratory. The outer conductor of the decelerator and the rf coupler housing were both machined out of oxygen-free electronic copper. The two sections, along with the WR112 output rectangular waveguide and 3-3/8 inches stainless steel flanges [21] were then brazed together at the Advanced Photon Source machine shop. The flange and the gasket at the rf output port were specially designed so that highpower rf signal can be transmitted while high vacuum is

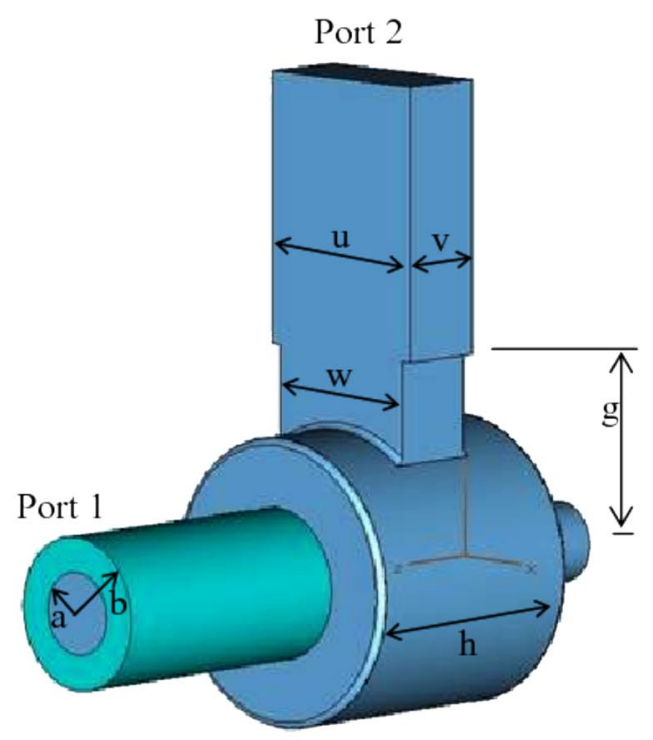

(a)

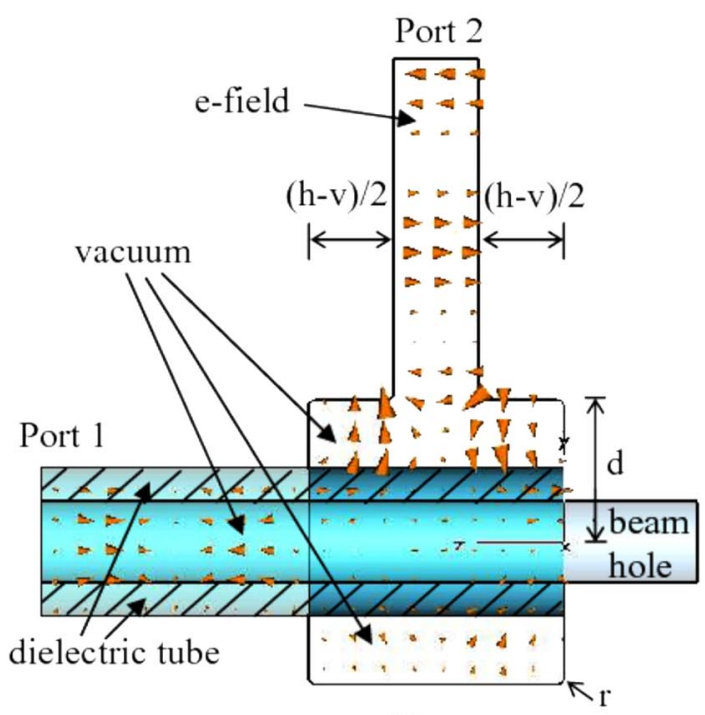

(b)

FIG. 6. (Color) CST geometry of the $\mathrm{TM}_{01}-\mathrm{TE}_{10}$ coupler with the background material set to the perfect electrical conductor boundary condition $(a=6.02 \mathrm{~mm}, \quad b=11.17 \mathrm{~mm}, \quad d=$ $21.30 \mathrm{~mm}, g=32.43 \mathrm{~mm}, h=38.32 \mathrm{~mm}, r=1.00 \mathrm{~mm}, u=$ $28.50 \mathrm{~mm}, v=12.62 \mathrm{~mm}, w=25.00 \mathrm{~mm}$ ). (a) Full view; (b) cutplane.

maintained. The configuration used for the low-power rf measurements of the power extractor is shown in Fig. 7.

A TEM-TM $\mathrm{TM}_{01}$ mode launcher was designed to convert the TEM mode to the $\mathrm{TM}_{01}$ mode for the dielectric-loaded waveguide. This is needed to couple the rf power from an HP8510C network analyzer, through the coax line, and into the dielectric-loaded waveguide. The mode launcher consists of a center pin with a disk on the tip, and a grounded copper plug inserted into the copper sleeve. The diameter of the disk, $d p$, is $3.24 \mathrm{~mm}$, the thickness of the disk, $t p$, is $2 \mathrm{~mm}$, and the distance between the disk and the grounded 


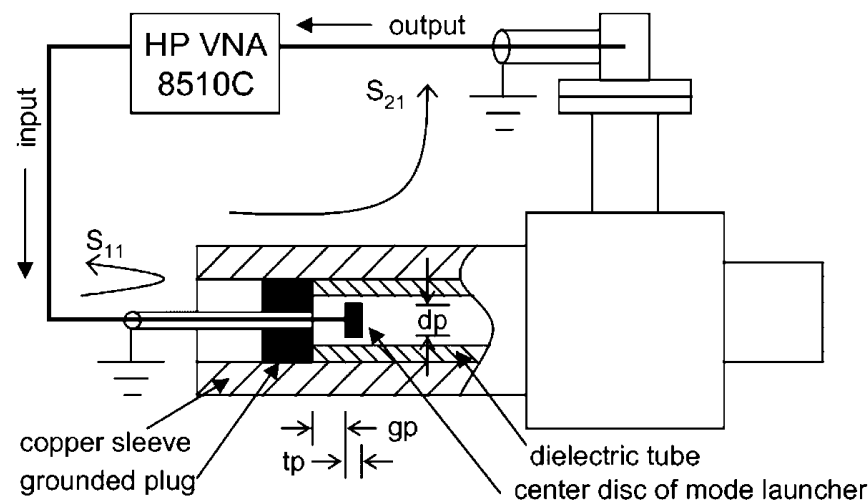

FIG. 7. Setup used for the rf benchtop measurement of the decelerator. A homemade mode launcher $(d p=3.24 \mathrm{~mm}, t p=$ $2 \mathrm{~mm}, g p=4.67 \mathrm{~mm}$ ) couples power into the dielectric-loaded waveguide, through the $\mathrm{TM}_{01}-\mathrm{TE}_{10}$ coupler, and into a commercial waveguide-to-coax adapter.

plug, $g p$, is $4.67 \mathrm{~mm}$. The simulated $\mathrm{S}_{21}$ of this mode launcher is $-0.036 \mathrm{~dB}$ at $7.8 \mathrm{GHz}$. It is better than $-0.1 \mathrm{~dB}$ in the range between 7.57 and $8.06 \mathrm{GHz}$, and better than $-1 \mathrm{~dB}$ in the range between 7.36 and $8.41 \mathrm{GHz}$. For comparison, the simulated $S_{21}$ of the rf output coupler (described in Sec. III B) is $-0.23 \mathrm{~dB}$ at $7.8 \mathrm{GHz}$, and better than $-1 \mathrm{~dB}$ in the range between 7.71 and $7.94 \mathrm{GHz}$. It can be seen that the bandwidth of the mode launcher is much wider than that of the rf output coupler, thus the insertion loss of the mode launcher is negligible. At the output side,

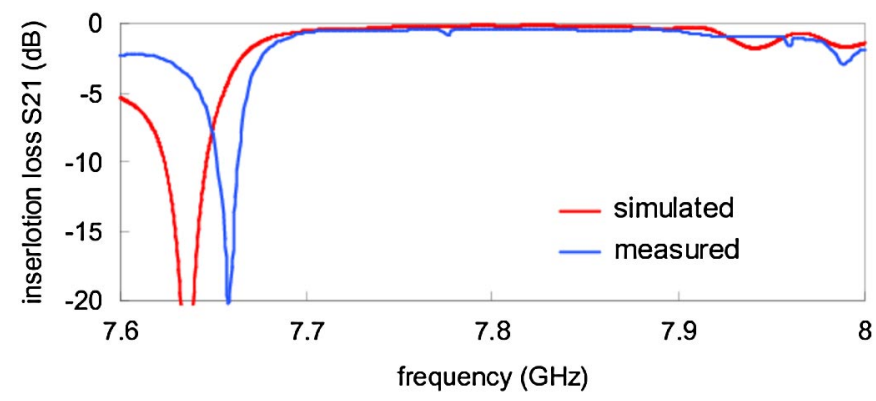

(a)

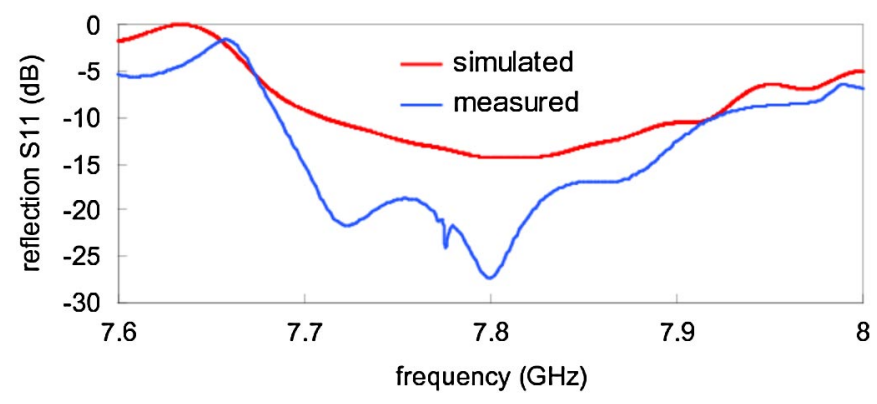

(b)

FIG. 8. (Color) Comparison of the measured and simulated transmission $S_{21}$ and reflection $S_{11}$ coefficients (mode launcher-rf output coupler assembly). (a) Insertion loss $\mathrm{S}_{21}$; (b) reflection $S_{11}$. a commercial WR112 waveguide-coax adapter [22] is used to convert the $\mathrm{TE}_{10}$ mode to the TEM mode for the output cable.

The S-parameters of the combined mode launcher and power-extractor system were measured and simulated; results (Figs. 8) show good agreement. Figure 8(a) shows that the system has a measured insertion loss $\mathrm{S}_{21}=$ $-0.41 \mathrm{~dB}$ at $7.8 \mathrm{GHz}$, corresponding to a power coupling efficiency $91 \%$. Figure 8(a) also shows that from 7.69 to $7.96 \mathrm{GHz} \mathrm{S_{21 }}$ is better than $-1 \mathrm{~dB}$, and from 7.67 to $7.99 \mathrm{GHz} S_{21}$ is better than $-3 \mathrm{~dB}$. The measured $S_{11}$ is better than the simulated value, and the small peak at $7.77 \mathrm{GHz}$ may be due to the fact the dielectric tube is not perfectly on axis. The peak at $7.65 \mathrm{GHz}$ in Fig. 8(a) is due to the resonance inside the metallic tube (inner radius $d$ ) in Fig. 6(b). To increase the bandwidth, adding a tapered section to the dielectric-loaded waveguide would help at the expense of increasing the length. However, when longer rf pulses are generated ( $>10 \mathrm{~ns}$ ), this bandwidth is not a critical limitation anymore.

\section{BEAM TESTS}

In this section, we briefly introduce the AWA facility, where the high-power rf tests were conducted and describe the experimental setup used. We then give a detailed description of the rf power generation measurements, first with a single bunch and second with a bunch train.

\section{A. AWA beam line and facility}

A simplified diagram of the AWA beam line (Fig. 9) shows only the beam line elements that were relevant to this experiment. It consists of a $1.3 \mathrm{GHz}$ photocathode gun and linac that delivers high-charge $(q: 1 \mathrm{nC}-100 \mathrm{nC})$, short bunch $\left(\sigma_{z}: 1.5-2.5 \mathrm{~mm}\right), 14 \mathrm{MeV}$ electron bunches. Relevant diagnostics include: charge measurement (integrating current transformer, ICT) and beam profile monitors (YAG:Ce) that are placed upstream and downstream of the device under test (DUT). The charge is easily varied by changing the intensity of the laser beam on the photocathode.

The AWA beam line components are tuned to maximize charge transmission through the power extractor. Quadrupole magnets and steering magnets were used to adjust the focusing and direction of the incoming drive bunch to maximize the charge transmitted through the power extractor. At high charge, the transmission is limited due to contributions from both space charge and emittance,

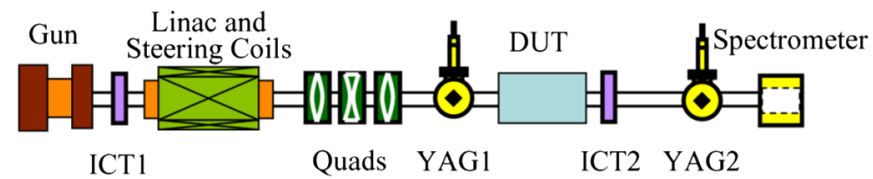

FIG. 9. (Color) Simplified diagram of the AWA beam line used during the beam tests. 
and usually the bunch is scrapped at the entrance to the DUT. The YAG and ICT diagnostics are used to both monitor and help maximize the transmission, where ICT1 records the charge emitted from the gun, and ICT2 records the charge transmitted through the power extractor.

Currently, the facility is capable of operating with single-bunch charges over $100 \mathrm{nC}$, while with multiple bunches, it operates at lower charge per bunch (1-10 nC) due to the relatively low quantum efficiency of the $\mathrm{Mg}$ photocathode, and laser power losses due to the multiple beam splitting technology. In the near future, a high quantum efficiency photocathode $\left(\mathrm{Cs}_{2} \mathrm{Te}\right)$ will be installed, which will enable high-charge bunch trains of $50 \mathrm{nC}$ per bunch and up to 64 consecutive bunches.

\section{B. Beam measurements}

The power extractor was tested with both a single bunch and a bunch train at the AWA facility. The power extractor electron beam test setup is schematically shown in Fig. 10. It is installed in the region labeled DUT in Fig. 9. In the multiple bunch setup, a spectrometer is used to monitor the bunch energy of each bunch in the train individually. This ensures that all particles were launched from the rf photoinjector at the same rf phase which ensure that the time between consecutive bunches is constant. As described in the previous sections, the drive-bunch excited rf power is extracted to a WR112 waveguide through the $\mathrm{TM}_{01}-\mathrm{TE}_{10}$ rf coupler. The waveguide is equipped with an ultrahigh vacuum (UHV) compatible bidirectional coupler $(-64.6 \mathrm{~dB})$ to measure both forward and backward TW power. The detected signals are recorded by a $15 \mathrm{GHz}$ bandwidth (sampling rate: $40 \mathrm{GS} / \mathrm{s}$ ) real-time digital oscilloscope [23]. Although an rf load is desired to absorb the extracted rf power and minimize reflections, such a highpower UHV load is not commercially available at $7.8 \mathrm{GHz}$. As an alternative, a long waveguide was used to delay the rf signal reflection by more than $14 \mathrm{~ns}$, thus the signal could be separated in time from its reflection on the oscilloscope. The vacuum pressure inside the power extractor reached $7 \times 10^{-9}$ Torr after ten days of pumping.

\section{Single-bunch tests}

During the single-bunch tests we studied the output power versus charge over a range from 10 to $66 \mathrm{nC}$. The

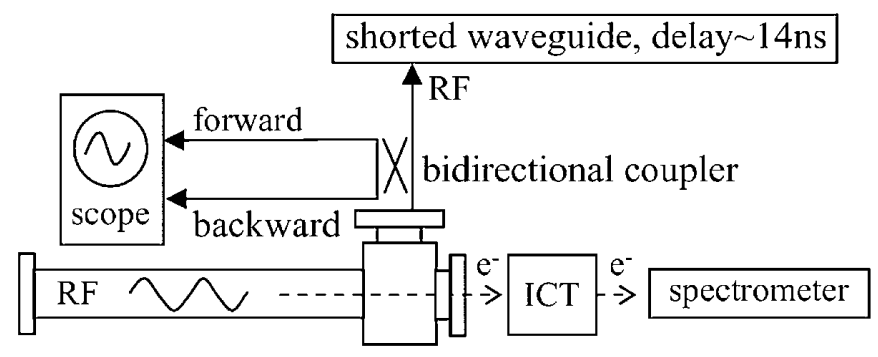

FIG. 10. Experimental setup for the beam tests.

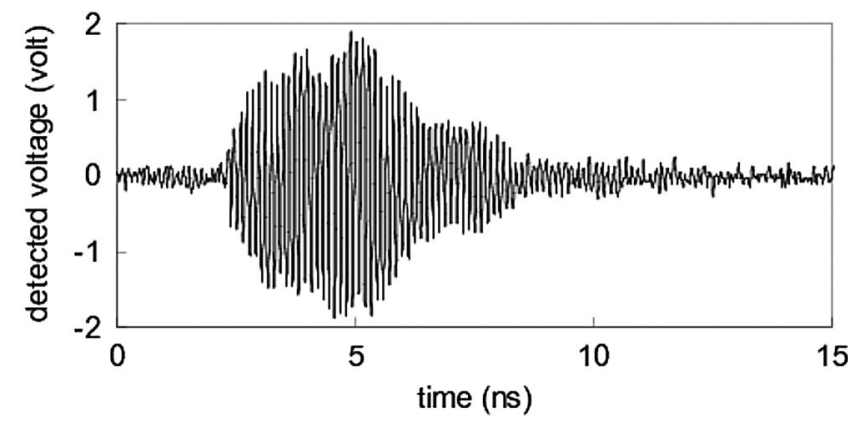

(a)

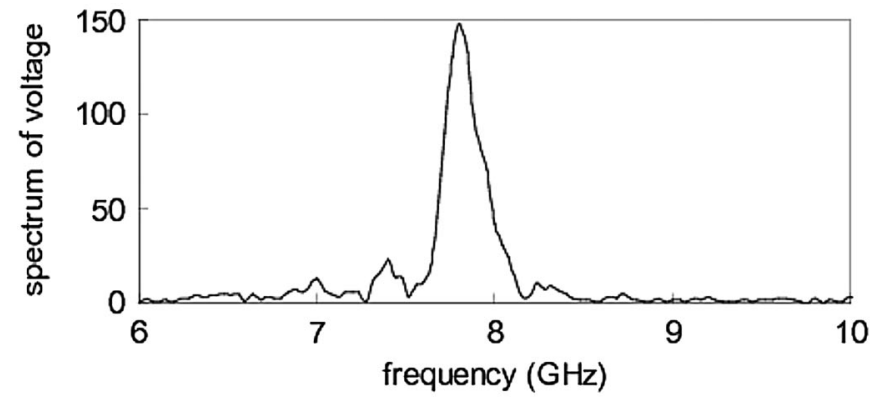

(b)

FIG. 11. Extracted rf signal measured on the Tektronix Oscilloscope during a beam test. Voltage signal excited by a $66 \mathrm{nC}$ single bunch (a) and its spectrum (b).

extracted rf signal was measured with Tektronix oscilloscope, and the charge transmitted through the power extractor was measured with ICT2. Figure 11(a) shows the measured voltage and Fig. 11(b) shows the corresponding frequency spectrum after digital Fourier transform of the voltage signal. The frequency of the measured signal is $7.8 \mathrm{GHz}$ indicating a good agreement with theory and simulations. The pulse length is $1.7 \mathrm{~ns}$, smaller than 2.2 ns simulated with MAFIA (Sec. III A), and smaller than the pulse length given by Eq. (3), 2.9 ns; this is due to the bandwidth limitation from the coupler. The power generated by different charge is plotted in Fig. 12. From the

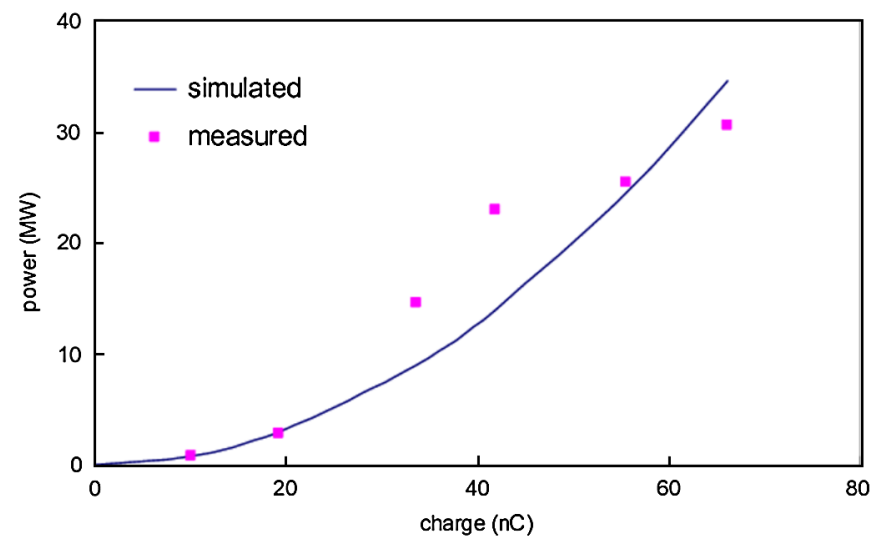

FIG. 12. (Color) Measured and simulated power generated vs charge. 
figure we can see that the measured results agree well with simulation, and $30 \mathrm{MW}$ of power is generated when the driving charge is $66 \mathrm{nC}$. The maximum single-bunch charge transmitted through the decelerator was $66 \mathrm{nC}$, as measured by ICT2.

\section{Bunch-train tests}

Bunch-train tests clearly demonstrated field superposition by multiple bunches. In the experiment, a laser beam splitter set is used to generate four consecutive electron bunches (the least bunches needed to reach the power saturation level), with a 769 ps time spacing between them, corresponding to a bunch frequency of $1.3 \mathrm{GHz}$. Various arrangements of bunch trains can be made by blocking various laser paths. Each bunch is individually checked with the spectrometer to ensure their launching phase in the gun (and hence time spacing) is the same, and with the ICT to ensure their charge is also the same. As shown in Figs. 13(a)-13(c), voltage signals are obtained from excitations by a single bunch, the first two bunches, and all four bunches. They clearly show that when more bunches are added in phase, not only the magnitude of the voltage signal gets larger, the pulse also gets longer. Figure 13(d) shows the spectrum of the signal in
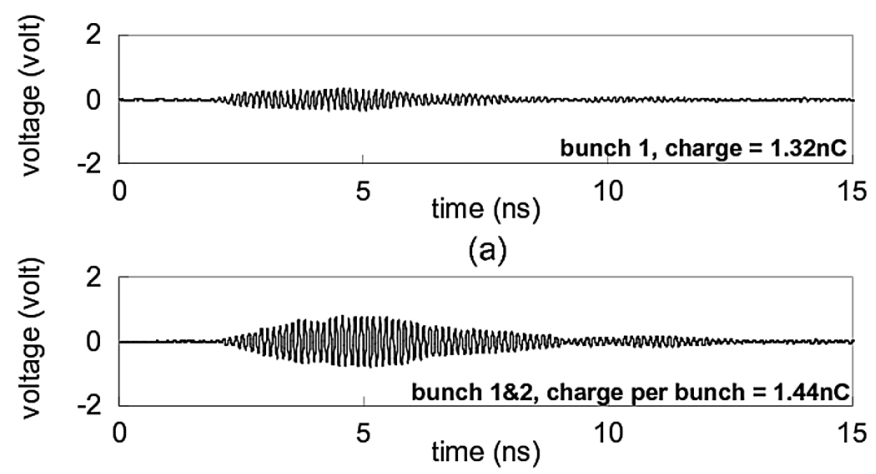

(b)

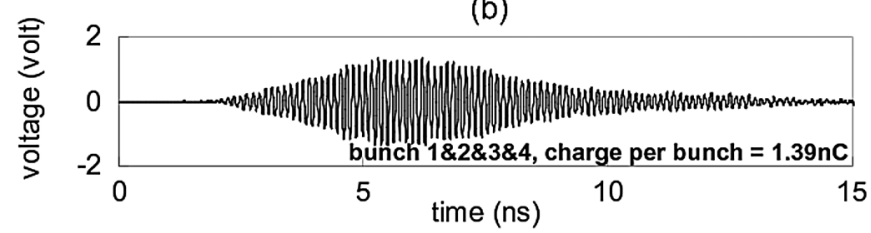

(c)

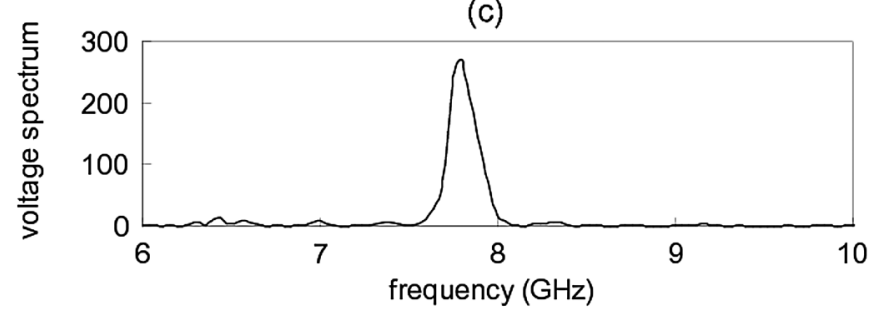

(d)

FIG. 13. Voltage measurements of the longitudinal electric field amplitude excited during beam tests: (a) by the first bunch only; (b) by the first and second bunches; (c) by all four bunches; (d) frequency spectrum of (c).
Fig. 13(c), from which we can see the center frequency is $7.8 \mathrm{GHz}$. Finally, after carefully taking into account all the attenuation, we estimate that the peak power from the four bunches is $0.19 \mathrm{MW}$, close to the simulated value $0.22 \mathrm{MW}$.

\section{SUMMARY}

A $7.8 \mathrm{GHz}$ dielectric-based power extractor has been theoretically and numerically analyzed, designed, fabricated, and tested at the AWA facility. Measurement results are in good agreement with both theory and numerical simulations. $30 \mathrm{MW}$ of $\mathrm{rf}$ power with a pulse length of approximately $1.7 \mathrm{~ns}$ was extracted and measured in the single-bunch test without any indication of breakdown. Field superposition due to a train of 4 bunches was also clearly demonstrated.

In the future, we plan to send a longer, higher charge bunch train through the power extractor to generate a longer, higher power rf output pulse. When the AWA upgrade is completed, the bunch train of up to $50 \mathrm{nC} \times$ 64 bunches will be able to generate $280 \mathrm{MW}$ of rf power at an $\mathrm{rf}$ pulse length of $50 \mathrm{~ns}$ in this structure.

\section{ACKNOWLEDGMENTS}

This work is supported by the U.S. Department of Energy under Contract No. DE-AC02-06CH11357.

\section{APPENDIX: DERIVATIONS OF THE FORMULAS FOR PULSED RF POWER GENERATED BY ULTRARELATIVISTIC DRIVE BUNCHES}

In this Appendix, detailed derivations for the rf power level and rf pulse length expressions of Sec. II are shown.

\section{Drive bunch train}

When a charged particle bunch (drive bunch) passes through the decelerator, it excites a wakefield which, in turn, carries a TW power $P_{w}$. We define the direction of the TW as $z+$, the entrance of the decelerator as $z=0$, and its exit as $z=L$. In the absence of an external rf power source all $\mathrm{rf}$ power comes from the wakefield of the drive bunch. For each mode, the power decreases along the length of the structure as

$$
\frac{d P_{w}}{d z}=-2 \alpha_{0} P_{w}
$$

where $\alpha_{0}$ is the field attenuation per unit length. In the presence of an infinitely long charged particle bunch train with bunch spacing $T_{b}$ and bunch charge $q$, a second term representing the power increase per unit length due to the wakefield must be added to Eq. (A1) [24],

$$
\frac{d P_{w}}{d z}=\frac{q E_{a}}{T_{b}}-2 \alpha_{0} P_{w}
$$

where it is assumed that all the particles experience the same deceleration gradient $E_{a}$. (i.e. the bunch length is 
short compared to the mode wavelength, $\sigma_{z} \ll \lambda_{\mathrm{rf}}$, so that all particles within the bunch experience the same rf phase).

For nonzero bunch length, the particles within a bunch experience different deceleration gradients less than or equal to the on-crest gradient $E_{a}$ and therefore a bunch form factor $\Phi$ is added to Eq. (A2),

$$
\frac{d P_{w}}{d z}=\frac{q E_{a}}{T_{b}} \Phi-2 \alpha_{0} P_{w}
$$

where for an ultrarelativistic bunch, $\Phi$ is expressed as $[18,25]$

$$
\Phi=\left|\frac{1}{q} \int_{-\infty}^{+\infty} f(z) e^{-j k_{z} z} d z\right|,
$$

$f(z)$ is the spatial charge distribution for one bunch, and $k_{z}$ is the longitudinal wave number of the rf field. In other words, $\Phi$ is just the magnitude of the Fourier transform of the charge distribution function, $f(z)$. For simplicity, we have dropped the explicit $k_{z}$ dependence $\left(\Phi\left(k_{z}\right)=\Phi\right)$ since the above equations apply to each mode individually. It is straightforward to show that for a Gaussian spatial bunch distribution $f(z)=\frac{q}{\sigma_{z} \sqrt{2 \pi}} \exp \left(-\frac{z^{2}}{2 \sigma_{z}^{2}}\right)$, with rms length $\sigma_{z}$, that the form factor is given by

$$
\Phi=\exp \left[-\left(k_{z} \sigma_{z}\right)^{2} / 2\right] .
$$

Following the derivation by Whittum [24], for a constant impedance structure, we first eliminate $P_{w}$ from Eq. (A3) using Eq. (A6):

$$
P_{w}=\frac{E_{a}^{2} v_{g}}{4 k_{L}}
$$

substituting Eq. (A6) into Eq. (A3) we can get

$$
\begin{gathered}
\frac{d}{d z}\left(\frac{E_{a}^{2} v_{g}}{4 k_{L}}\right)=\frac{q \Phi E_{a}}{T_{b}}-\frac{E_{a}^{2} v_{g} \alpha_{0}}{2 k_{L}} \\
\frac{d E_{a}}{d z}+\alpha_{0} E_{a}=\frac{2 q k_{L} \Phi}{T_{b} v_{g}} \\
\frac{d}{d z}\left(E_{a} e^{\alpha_{0} z}\right)=\frac{2 q k_{L} \Phi}{T_{b} v_{g}} e^{\alpha_{0} z} \\
E_{a}(z)=e^{-\alpha_{0} z}\left[E_{a}(0)+\frac{2 q k_{L} \Phi}{T_{b} v_{g}} \int_{0}^{z} e^{\alpha_{0} \tau} d \tau\right] .
\end{gathered}
$$

We can further simplify this expression by realizing that the bunch must travel some distance before losing energy, or $E_{a}(0)=0$. Therefore, Eq. (A10) can be rewritten as

$$
E_{a}(z)=\frac{2 q k_{L}}{T_{b} v_{g} \alpha_{0}}\left(1-e^{-\alpha_{0} z}\right) \Phi .
$$

At the exit of the decelerator $(z=L)$, the longitudinal electrical field amplitude is

$$
E_{a t}=E_{a}(L)=\frac{2 q k_{L}}{T_{b} v_{g} \alpha_{0}}\left(1-e^{-\alpha_{0} L}\right) \Phi .
$$

Finally, substituting Eq. (A12) into Eq. (A6), one can get the power level at the end of the deceleration waveguide shown in Eq. (A13), rewritten as

$$
P_{t}=q^{2} \frac{k_{L}}{v_{g}}\left(\frac{1-e^{-\alpha_{0} L}}{\alpha_{0} T_{b}}\right)^{2} \Phi^{2} .
$$

Furthermore, when the decelerator is lossless $\left(\alpha_{0}=0\right)$, Eq. (A13) can be modified as Eq. (A14):

$$
P_{t}=q^{2} \frac{k_{L}}{v_{g}}\left(\frac{L}{T_{b}}\right)^{2} \Phi^{2}
$$

\section{B. Single drive bunch}

The power level generated by a single bunch can be obtained by modifying Eq. (A14) if we arrange for the net rf pulse to be constant. This can be done by changing the decelerator length, $L$, so that the single-bunch pulse duration, $\tau_{s}$, is exactly the same as the bunch spacing $T_{b}$. In this case, the individual rf pulses exit the decelerator stacked one after the other, without overlap or a gap between them, according to illustration in Fig. 4. Therefore, the power level calculated from Eq. (A14) will be the same as that excited by a single bunch. In other words, by replacing $T_{b}$ with $\tau_{s}$ in Eq. (A14), we obtain the power level at the exit of a lossless decelerator excited by a single bunch

$$
P_{s}=q^{2} k_{L} v_{g}\left(\frac{1}{1-\beta_{g}}\right)^{2} \Phi^{2} \quad\left(0 \leq t \leq \tau_{s}\right) .
$$

For a lossy $\left(\alpha_{0} \neq 0\right)$ structure, Eq. (A15) can be modified to yield Eq. (A16):

$$
P_{s}(t)=q^{2} k_{L} v_{g}\left(\frac{1}{1-\beta_{g}}\right)^{2} \Phi^{2} e^{-2 \alpha_{0} v_{g} t} \quad\left(0 \leq t \leq \tau_{s}\right),
$$

where $t=0$ is the moment the bunch exits the decelerator, i.e., the moment the head of the excited rf pulse exits the decelerator.

[1] L. Evan, Particle Acceleration Conference, Albuquerque (IEEE, New York, 2007), p. 1.

[2] International Linear Collider Reference Design Report, 2007.

[3] A. M. Sessler, AIP Conf. Proc. 91, 154 (1982).

[4] H. Braun et al., CERN-CLIC Note 364.

[5] K. Kübner, XVth International Conference on High Energy Accelerators, Hamburg, 1992, p. 791.

[6] B. Danly, AIP Conf. Proc. 335, 25 (1995).

[7] W. Gai and P. Schoessow, Nucl. Instrum. Methods Phys. Res., Sect. A 459, 1 (2001). 
[8] A. M. Sessler and S. S. Yu, Phys. Rev. Lett. 58, 2439 (1987).

[9] W. Gai, M.E. Conde, R. Konecny, J. G. Power, P. Schoessow, X. Sun, and P. Zou, AIP Conf. Proc. 569, 287 (2001).

[10] M. E. Conde et al., AIP Conf. Proc. 877, 657 (2006).

[11] P. Zou, W. Gai, R. Konecny, X. Sun, T. Wong, and A. Kanareykin, Rev. Sci. Instrum. 71, 2301 (2000).

[12] E. Chjonacki, W. Gai, C. Ho, R. Konecny, S. Mtingwa, J. Norem, M. Rosing, P. Schoessow, and J. Simpson, J. Appl. Phys. 69, 6257 (1991).

[13] E. Chojnacki, W. Gai, P. Schoessow, and J. Simpson, Particle Acceleration Conference, San Francisco (IEEE, New York, 1991), p. 2557.

[14] M.E. Conde, W. Gai, R. Konecny, J. G. Power, P. Schoessow, and P. Zou, AIP Conf. Proc. 472, 626 (1999).

[15] D. Newsham et al., Particle Acceleration Conference, Portland, Oregon (IEEE, New York, 2003), p. 1156.
[16] T. Wangler, RF Linear Accelerator (John Wiley \& Sons, Inc., New York, 1998), Sec. 10.2.

[17] CST MICROWAVE STUDIO Version 5.0.0, developed by Computer Simulation Technology.

[18] D. Newsham, D. Newsham, and A. Smirnov, AIP Conf. Proc. 647, 484 (2002).

[19] A. Smirnov, Y. Luo, R. Yi, and D. Yu, Particle Acceleration Conference, Knoxville, Tennessee (IEEE, New York, 2005), p. 4078.

[20] CST MAFIA version 4.0, developed by Computer Simulation Technology.

[21] A\&N Coperation, Part No. 338-000-ANL.

[22] Space Machine \& Engineering Corp, Part No. WCA112.B5MF.CN.

[23] Tektronix Inc, model number TDS6154C.

[24] D. Whittum, Report No. SLAC-PUB-7802, 1998, Sec. 4.2.

[25] P. B. Wilson, Report No. SLAC-PUB-2884, 1991, Sec. 3.3. 